\title{
Temozolomide treatment can improve overall survival in aggressive pituitary tumors and pituitary carcinomas
}

\author{
Hélène Lasolle ${ }^{1,2}$, Christine Cortet ${ }^{3}$, Fréderic Castinetti ${ }^{4}$, Lucie Cloix ${ }^{5}$, Philippe Caron $^{6}$, Brigitte Delemer7, $^{7}$ \\ Rachel Desailloud ${ }^{8}$, Christel Jublanc ${ }^{9}$, Christine Lebrun-Frenay ${ }^{10}$, Jean-Louis Sadoul ${ }^{11}$, Luc Taillandier ${ }^{12}$, \\ Marie Batisse-Lignier ${ }^{13}$, Fabrice Bonnet ${ }^{14}$, Nathalie Bourcigaux ${ }^{15}$, Damien Bresson ${ }^{16}$, Olivier Chabre ${ }^{17}$, \\ Philippe Chanson 18,19, Cyril Garcia ${ }^{20}$, Magalie Haissaguerre ${ }^{21}$, Yves Reznik ${ }^{22}$, Sophie Borot ${ }^{23}$, Chiara Villa ${ }^{24,25}$, \\ Alexandre Vasiljevic ${ }^{26}$, Stephan Gaillard ${ }^{27}$, Emmanuel Jouanneau ${ }^{2,28}$, Guillaume Assié ${ }^{29}$ and Gérald Raverot ${ }^{1,2}$ \\ ${ }^{1}$ Fédération d'Endocrinologie, Groupement Hospitalier Est, Hospices Civils de Lyon, Bron, France, ${ }^{2}$ Université Lyon 1, Lyon, France, ${ }^{3}$ Service \\ d'Endocrinologie, Hôpital Claude Huriez, CHRU de Lille, Lille, France, ${ }^{4}$ Service d'Endocrinologie, Hôpital de La Timone, CHU de Marseille, Marseille, \\ France, ${ }^{5}$ Service d'Endocrinologie, Hôpital Bretonneau, CHRU de Tours, Tours, France, ${ }^{6}$ Service d'Endocrinologie, Hôpital Larrey, CHU de Toulouse, \\ Toulouse, France, 7 Service d'Endocrinologie, Hôpital Robert Debré, CHU de Reims, Reims, France, ${ }^{8}$ Service d'Endocrinologie, Hôpital Nord, CHU \\ d'Amiens-Picardie, Amiens, France, 9 Service d'Endocrinologie, Hôpital de La Pitié-Salpêtrière, Assistance Publique des Hôpitaux de Paris, Paris, France, \\ ${ }^{10}$ Service de Neurologie, Unité de Neuro-oncologie, Hôpital Pasteur 2, CHU de Nice, Nice, France, ${ }^{11}$ Service d'Endocrinologie, Hôpital L'Archet, CHU \\ de Nice, Nice, France, ${ }^{12}$ Service de Neurologie, CHU de Poitiers, Poitiers, France, ${ }^{13}$ Service d'Endocrinologie, Hôpital Gabriel Montpied, CHU de \\ Clermont-Ferrand, Clermont-Ferrand, France, ${ }^{14}$ Service d'Endocrinologie, CHU de Rennes, Rennes, France, ${ }^{15}$ Service d'Endocrinologie, Hôpital Saint \\ Antoine, Assistance Publique des Hôpitaux de Paris, Paris, France, ${ }^{16}$ Service de Neurochirurgie, Hôpital Lariboisière, Assistance Publique des Hôpitaux \\ de Paris, Paris, France, ${ }^{17}$ Service d'Endocrinologie, CHU de Grenoble-Alpes, La Tronche, France, ${ }^{18}$ Service d'Endocrinologie et des Maladies de la \\ Reproduction, Hôpital de Bicêtre, Assistance Publique-Hôpitaux de Paris, Hôpitaux Universitaires Paris-Sud, Le Kremlin Bicêtre, France, \\ ${ }^{19}$ INSERM 1185, Fac Med Paris Sud, Univ Paris-Sud, Université Paris-Saclay, Le Kremlin-Bicêtre, France, ${ }^{20}$ Service d'Endocrinologie, Hôpital d'Instruction \\ des Armées Bégin, Saint-Mandé, France, ${ }^{21}$ Service d'Endocrinologie, Hôpital Haut-Lévêque, CHU de Bordeaux, Pessac, France, \\ ${ }^{22}$ Service d'Endocrinologie, CHU de Caen, Caen, France, ${ }^{23}$ Service d'Endocrinologie, Hôpital Jean Minjoz, CHU \\ de Besançon, Besançon, France, ${ }^{24}$ Service d'Anatomie et Cytologie Pathologiques, Hôpital Foch, Suresnes, France, \\ ${ }^{25}$ INSERM U1016 CNRS UMR 8104, Paris, France, ${ }^{26}$ Centre de Pathologie Est, Groupement Hospitalier Est, Hospices Civils \\ de Lyon, Bron, France, ${ }^{27}$ Service de Neurochirurgie, Hôpital Foch, Suresnes, France, ${ }^{28}$ Service de Neurochirurgie, Hôpital \\ Neurologique, Hospices Civils de Lyon, Bron, France, and ${ }^{29}$ Department of Endocrinology, Institut Cochin, INSERM \\ U1016, CNRS UMR8104, Paris Descartes University, Assistance Publique Hôpitaux de Paris, Hôpital Cochin, Paris, France \\ Correspondence \\ should be addressed \\ to G Raverot \\ Email \\ gerald.raverot@chu-lyon.fr
}

\section{Abstract}

Objectives: Only few retrospective studies have reported an efficacy rate of temozolomide (TMZ) in pituitary tumors (PT), all around $50 \%$. However, the long-term survival of treated patients is rarely evaluated. We therefore aimed to describe the use of TMZ on PT in clinical practice and evaluate the long-term survival.

Design: Multicenter retrospective study by members of the French Society of Endocrinology.

Methods: Forty-three patients (14 women) treated with TMZ between 2006 and 2016 were included. Most tumors were corticotroph $(n=23)$ or lactotroph $(n=13)$, and 14 were carcinomas. Clinical/pathological characteristics of PT, as well as data from treatment evaluation and from the last follow-up were recorded. A partial response was considered as a decrease in the maximal tumor diameter by more than $30 \%$ and/or in the hormonal rate by more than $50 \%$ at the end of treatment. Results: The median treatment duration was 6.5 cycles (range 2-24), using a standard regimen for most and combined radiotherapy for six. Twenty-two patients $(51.2 \%)$ were considered as responders. Silent tumor at diagnosis was associated with a poor response. The median follow-up after the end of treatment was 16 months (0-72). Overall survival was significantly higher among responders $(P=0.002)$; however, ten patients relapsed 5 months $(0-57)$ after the end of TMZ treatment, five in whom TMZ was reinitiated without success.

Discussion: Patients in our series showed a $51.2 \%$ response rate to TMZ, with an improved survival among responders despite frequent relapses. Our study highlights the high variability and lack of standardization of treatment protocols.

European Journal of Endocrinology (2017) 176, 769-777
두 2017 European Society of Endocrinology Printed in Great Britain
Published by Bioscientifica Ltd. 


\section{Introduction}

Pituitary tumors (PT) represent $10-15 \%$ of intra-cranial tumors (1). While most are benign and can be controlled by current therapeutics, some show a lack of sensitivity to a combination of therapies or recur during follow-up and are considered as aggressive with unclear epidemiology (2). For those with atypical morphological features suggestive of aggressive behavior (3) and accounting for 2.7-15\% of pituitary tumors (4), the World Health Organization (WHO) proposed the formulation 'atypical tumors' with 'uncertain malignancy'. Other features such as invasion can be predictive of aggressiveness (5). Lastly, $0.2 \%$ of pituitary tumors are associated with distant metastasis (most frequently in central nervous system, liver and bones) with a mean survival of between 1 and 3 years (6).

Temozolomide (TMZ) is an alkylating chemotherapy derivative of dacarbazine with lipophilic properties allowing it to cross the blood-brain barrier. Its efficacy was first demonstrated in glioblastomas (7) before its successful use in advanced melanomas and neuroendocrine tumors (8). With $100 \%$ oral bioavailability, the standard regimen for administering $\mathrm{TMZ}$ consists in an oral daily dose of $150-200 \mathrm{mg} / \mathrm{m}^{2}$ body surface area for 5 days every 28 days. Alternative protocols based on radio-sensitizing properties have been proposed, such as the Stupp protocol, though no superiority has yet been demonstrated (9). The standard duration of treatment for glioblastoma is 6-12 months (10).

The first successful treatment of PT with TMZ concerned prolactin-secreting carcinomas, as reported in 2006 (11, 12 , 13). There have since been many reported cases of aggressive PT and carcinomas from all tumor subtypes presenting hormonal decrease and tumor shrinkage with TMZ treatment. More recently, eight retrospective studies on between 5 and 33 patients $(14,15,16,17,18,19,20$, 21) reported lower efficacies of $T M Z$ and underlined the absence of standardization in treatment administration protocols and response evaluation (22). The reported response rates ranged from 29 to $81 \%$ depending on the response criteria, and the overall survival was estimated by Losa et al. as 59.6\% at four years after TMZ initiation with a median follow-up of 41 months (14).

In glioblastomas, low expression of the protein MGMT, as evaluated by promoter methylation analysis, which induces gene silencing, is correlated with tumor response to TMZ (23). Indeed, this protein has been shown to remove the methyl group bound by TMZ on 06-guanine. In PT however, the predictive role of MGMT remains debated as its promoter methylation does not consistently correlate with its immunohistochemistry expression (24). Also, while some studies have reported a relation between low MGMT expression and tumor sensitivity to $\operatorname{TMZ}(15,17,25)$, others have found no such relation $(18,19,20)$. Such discrepancies alongside the difficulties encountered in interpreting per-center immunohistochemistry analyses mean that today, the therapeutic decision cannot be made based on MGMT analysis.

Nevertheless, TMZ remains the main therapeutic resource currently available for PT resistant to conventional treatment. Its use is however limited by the absence of a standardized protocol of administration and evaluation, and by the requirement for a long-term follow-up. Here we have endeavored to address these outstanding issues by retrospectively studying a large cohort with the aim of describing the use of TMZ to treat PT in France and evaluating its short- and long-term effects on overall survival.

\section{Patients and methods}

\section{Patients}

Members of the French Society of Endocrinology were surveyed with regards to their patients treated with TMZ for PT. A total of 43 patients (29 men and 14 women) were declared as treated with TMZ between 2006 and 2016 in 21 centers. Ten patients were included in previous studies though a longer follow-up is presented here for $8(18,26$, $27,28,29)$. The median age at diagnosis was 43 years, ranging from 13 to 76 years. Baseline characteristics are presented in Table 1 .

\section{Pituitary tumors}

Twenty-nine locally aggressive adenomas and 14 carcinomas were treated with TMZ. Carcinomas were diagnosed based on unique or multiple distant sites of metastasis, that were located in the central nervous system for most (11 meningeal, 4 brain parenchyma, 1 liver and 2 bones). The diagnosis was made before TMZ treatment initiation for 12 patients. The median time between tumor diagnosis and metastasis discovery was 7 years (from 2 to 17 years).

Tumor type was identified using histological examination or, when not available as in six noneoperated patients, by measurement of hormonal secretion. Corticotroph tumors (ACTH) were the most frequent $(n=23)$, followed by lactotroph (PRL) $(n=13)$, 
Table 1 Baseline characteristics among responders and non-responders.

\begin{tabular}{l} 
Characteristics \\
\hline$n$ \\
Male/female \\
Age at diagnosis (years) \\
Age at TMZ treatment (years) \\
Carcinomas, $n$ \\
Initially silent or silent, $n$ \\
Tumor type, $n$ \\
PRL \\
ACTH \\
GH \\
GH/PRL \\
Immunonegative \\
Histological features \\
P53, $n$ positive \\
Ki67 $>3 \%, n$ positive \\
Mitosis $>2 / 10$ HPF, $n$ positive \\
Grade $2 b, n$ \\
MGMT analysis \\
MGMT promoter methylation, $n$ positive \\
MGMT expression, \% ( $n=13$ ) \\
TMZ treatment \\
Time before treatment (years) \\
Concomitant RT, $n$ \\
'Stupp', $n$ \\
Duration, $n$ cycles ( $n=38$ ) \\
\hline
\end{tabular}

\begin{tabular}{c}
\hline All patients \\
\hline 43 \\
$29 / 14$ \\
$43(13-76)$ \\
$54(18-80)$ \\
14 \\
12 \\
13 \\
23 \\
3 \\
3 \\
1
\end{tabular}

\begin{tabular}{c}
\hline Responding \\
\hline 22 \\
$13 / 9$ \\
$51(13-76)$ \\
$54(18-80)$ \\
6 \\
2
\end{tabular}

\begin{tabular}{c}
\hline Non-responding \\
21 \\
$16 / 5$ \\
$40(18-63)$ \\
$55(26-75)$ \\
8 \\
10
\end{tabular}

\begin{tabular}{c}
\hline $\boldsymbol{P}$-value ${ }^{\mathrm{a}}$ \\
\hline NS \\
0.41 \\
NS \\
NS \\
$0.03^{*}$ \\
0.37
\end{tabular}

Quantitative data are presented as median (min-max).

\begin{tabular}{c}
$17 / 21$ \\
$25 / 31$ \\
$14 / 19$ \\
$20 / 23$ \\
$3 / 10$ \\
$18(0-100)$ \\
$6(0-23)$ \\
10 \\
6 \\
$6.5(2-24)$ \\
\hline
\end{tabular}

7
12
0
3
0
$5 / 8$
$12 / 14$
$4 / 6$
$7 / 9$
$2 / 4$

6
11
3
0

$9.5(0-100)$

$12 / 13$
$13 / 17$
$10 / 13$
$13 / 14$
$1 / 6$
$30(0-100)$
$10(0-23)$
3
1
$6(2-16)$

\begin{tabular}{l}
0.39 \\
NS \\
NS \\
NS \\
\\
NS \\
NS \\
\\
0.17 \\
0.76 \\
0.89 \\
$<0.001$ \\
\hline
\end{tabular}

alikelihood ratio tests of responders vs non-responders at multivariate analysis, except for duration of treatment (univariate analysis). *significant value.

somatotroph $(\mathrm{GH})(n=3)$, somato-lactotroph $(\mathrm{GH} / \mathrm{PRL})$ $(n=3)$ and finally one immunonegative tumor. Among 12 tumors that were silent at diagnosis (8 ACTH, 2 GH, 1 PRL, 1 immunonegative), seven became secreting during follow-up before TMZ initiation.

The proliferation criteria P53 expression, Ki67 expression $>3 \%$ and number of observed mitoses $>2 / 10$ HPF were positive in 17/21, 25/31 and 14/19 evaluated tumors respectively. They three pathological markers were missing for 11 tumors (six of whom had not been operated). Two criteria or more were available for 23 tumors. Twenty presented two positive proliferative criteria and were classified as grade $2 \mathrm{~b}(5)$.

Measurement of MGMT expression was available for 13 patients, without centralized analysis, while MGMT promoter methylation status was available for ten patients, and both were available for nine patients.

\section{Temozolomide treatment evaluation}

Age at treatment initiation as well as treatment dose and duration were recorded. Radiotherapy administered within four months before initiation of TMZ treatment was considered as concomitant.
Biological and radiological evaluations were trimestral. The response was evaluated either at the end of the treatment or at the last follow-up if ongoing. In the absence of a complete history of tumor course before TMZ treatment, stability was not considered as a positive response. A tumor response was defined as a decrease of more than $30 \%$ of the largest tumor diameter and a hormonal response as a $50 \%$ or more decrease in baseline hormone levels. The overall response was considered as positive in cases of a hormonal and/or a tumor response, and negative otherwise. Response data were available for all patients but only tumor response was evaluated for six patients (four silent tumors, one immunonegative tumor and one patient with missing hormonal data).

\section{Statistical analysis}

Data are presented as a median and range (minimum and maximum).

The association between response (positive or negative) and baseline/treatment characteristics was evaluated using logistic regression models, which were compared with likelihood ratio test (LRT). Characteristics which could be associated at univariate analysis $(P<0.20)$ 
were considered in multivariate analysis. Only these results are presented. Histological features were analyzed in a separate model because of abundant missing data. The duration of treatment was not considered in multivariate analysis due to it likely being influenced by response status.

Overall survival and relapse-free survival after the end of TMZ treatment were estimated using the Kaplan-Meier method. Survival was considered from the end of TMZ treatment and patients were censored at the date of last follow-up. Overall survival among responders and nonresponders were compared using a log-rank test (univariate analysis) and Cox model (multivariate analysis). Relapsefree survival among patients treated more and less than 12 cycles were compared using a log-rank test.

All analyses were performed using R software (http:// www.R-project.org/) and $P$ values $<5 \%$ were considered significant.

\section{Results}

\section{Temozolomide treatment indication}

Twelve patients received TMZ treatment because of distant metastasis. Locally aggressive adenomas at the time of treatment $(n=31)$ presented as a growing tumor despite surgical intervention $(n=8)$, radiation therapy $(n=1)$ or both $(n=19)$. Three inoperable patients had no surgery or radiation therapy (one non-secreting ACTH and two drug-resistant prolactinomas); two of them received a radiotherapy-temozolomide combined treatment. All secreting tumors showed resistance to medical treatment at the time of TMZ initiation $(n=38)$.

\section{Temozolomide treatment}

Patients received TMZ after a median of $6(0-23)$ years following tumor diagnosis and at a median age of 54 (18-80) years.

In most cases, the TMZ treatment regimen consisted of $150 \mathrm{mg} / \mathrm{m}^{2} /$ day during 5 days every 28 days. Patients displaying a good toleration of the treatment had their dose increased to $200 \mathrm{mg} / \mathrm{m}^{2} /$ day at the second cycle.

Six of the patients treated after 2011 underwent combined radiotherapy, receiving a daily dose of $75 \mathrm{mg} / \mathrm{m}^{2}$ associated with stereotactic radiotherapy during 6 weeks, followed by a standard TMZ administration protocol ( 6 cycles for 4 patients, then 12 and 17 cycles for the other two). Including those undergoing this 'Stupp' protocol, 10 patients underwent concomitant radiotherapy.
The treatment was ongoing in five patients at the last follow-up, after 3-24 cycles. Finished treatments had a median duration of 6.5 cycles (2-24) with a significant difference among responders and non-responders (respectively 12 (5-24) and 6 (2-16) cycles) (Fig. 1 and Table 1).

\section{Temozolomide tolerance}

Temozolomide treatment was globally well-tolerated with only one observed cessation due to side effects. The most frequent side effects were hematologic $(n=10)$, including isolated thrombocytopenia $(n=6)$, isolated lymphopenia $(n=2)$, bicytopenia $(n=2)$ and pancytopenia $(n=2)$. No gradations were collected however treatment could be continued at either the same dose or a decreased dose in all but one case. This patient presented a severe pancytopenia requiring treatment cessation and presented anew thrombocytopenia when treatment was started again. Ten patients reported asthenia that required dose decrease for two. Digestive disorders were reported in 10 patients (nausea, vomiting, abdominal pain and diarrhea). One patient presented severe headaches at every cycle, which were calmed by corticosteroids. One patient suffered tumoral and intraventricular hemorrhage during treatment, and another normal pressure hydrocephalus.

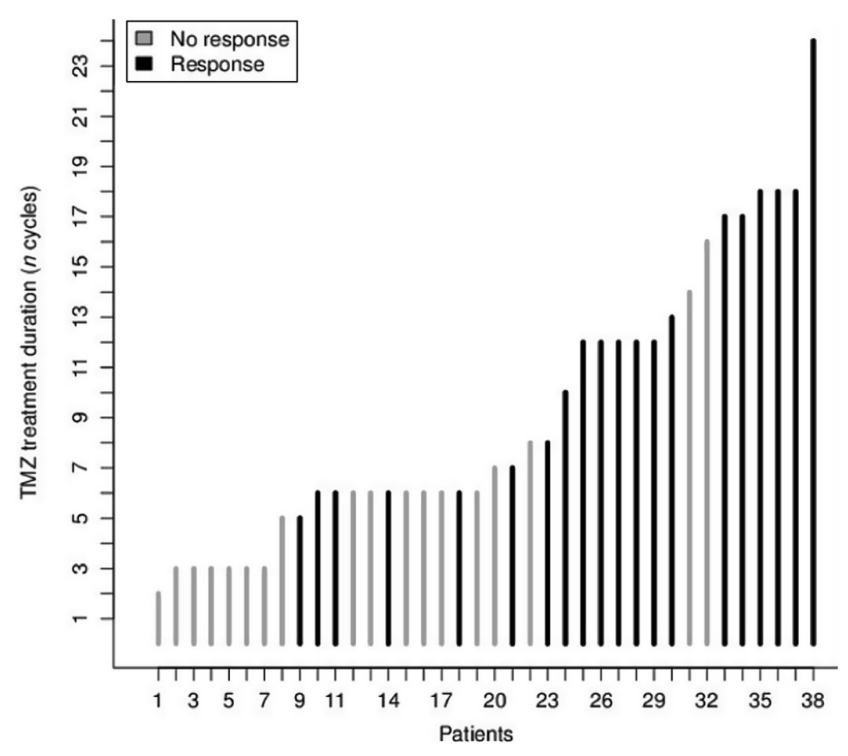

Figure 1

Histogram of duration of finished treatment according to response status. 


\section{Temozolomide efficacy}

We evaluated the efficacy of TMZ at the end of treatment for 38 patients and at the last administered cycle for five patients. Twenty-two patients were presented as responders (hormonal and/or tumor response) and 21 did not fulfill the response criteria.

Among the responders, 12 presented both hormonal and tumor responses, five a hormonal response only (three ACTH, one PRL/GH, one GH tumors) and four patients with a secreting tumor presented a tumor response only. Hormonal evaluation was missing for one patient presenting tumor shrinkage. Three secreting ACTH tumors showed a complete response.

Among the non-responders, 11 patients showed disease progression (hormone level or tumor diameter increasing after three (3-14) cycles) of whom two presented metastasis apparition, and ten patients were considered as stable. All non-secreting tumors at the time of treatment (two silent ACTH, one silent GH, one silent PRL and one immunonegative), and five out of the seven initially silent tumors, were non-responders.

Among the 22 patients considered as responders at the end of the treatment, 19 fulfilled the response criteria at the first evaluation conducted after 3-6 cycles. Surprisingly, one of these patients presented discordant evolution with initial hormonal and tumor response that evolved into a tumor response only, and increase in ACTH levels that no longer met response criteria. Moreover, two patients met hormonal criteria only after 3 cycles before showing both a hormonal and tumor response by the end of the cycles. One patient presented insufficient tumor and hormonal response initially before showing both.

At univariate analysis, tumor type, a secreting tumor at diagnosis, and short time interval between tumor diagnosis and TMZ initiation were significantly associated with a positive response to TMZ. 'Stupp' protocol or P53 expression showed a tendency toward an association with positive response though neither reached statistical significance. We found an initially silent tumor as the sole factor that remained associated with response status in multivariate analysis, being predictive of no response to treatment (odds ratio (OR): $0.13,95 \%$ CI: (0.01-0.81)). While MGMT expression levels tended to be lower among responders than non-responders (Fig. 2), neither MGMT expression nor its promoter methylation significantly correlated with TMZ response. Moreover, MGMT expression level measured with IHC did not significantly associate with promoter methylation presence $(P$-value Wilcoxon rank test 0.5) (Fig. 2).

\section{Long-term follow-up and survival}

Among the 38 patients no longer receiving TMZ treatment, the median follow-up after the end of treatment was 16 (0-72) months (18 (0-72) months among 20 responders and 14 (0-42) months among 18 non-responders) (Fig. 3).

Post TMZ treatment, the estimated overall median survival (95\% IC) among our patients was 40 months (16-44) (Fig. 4A) with an estimated survival probability of $51.3 \%(32.4-70.3)$ at 3 years. The median survival of 44 months (42-infinity) (95\% CI) among responders was significantly higher than the 16 months (9-25) found
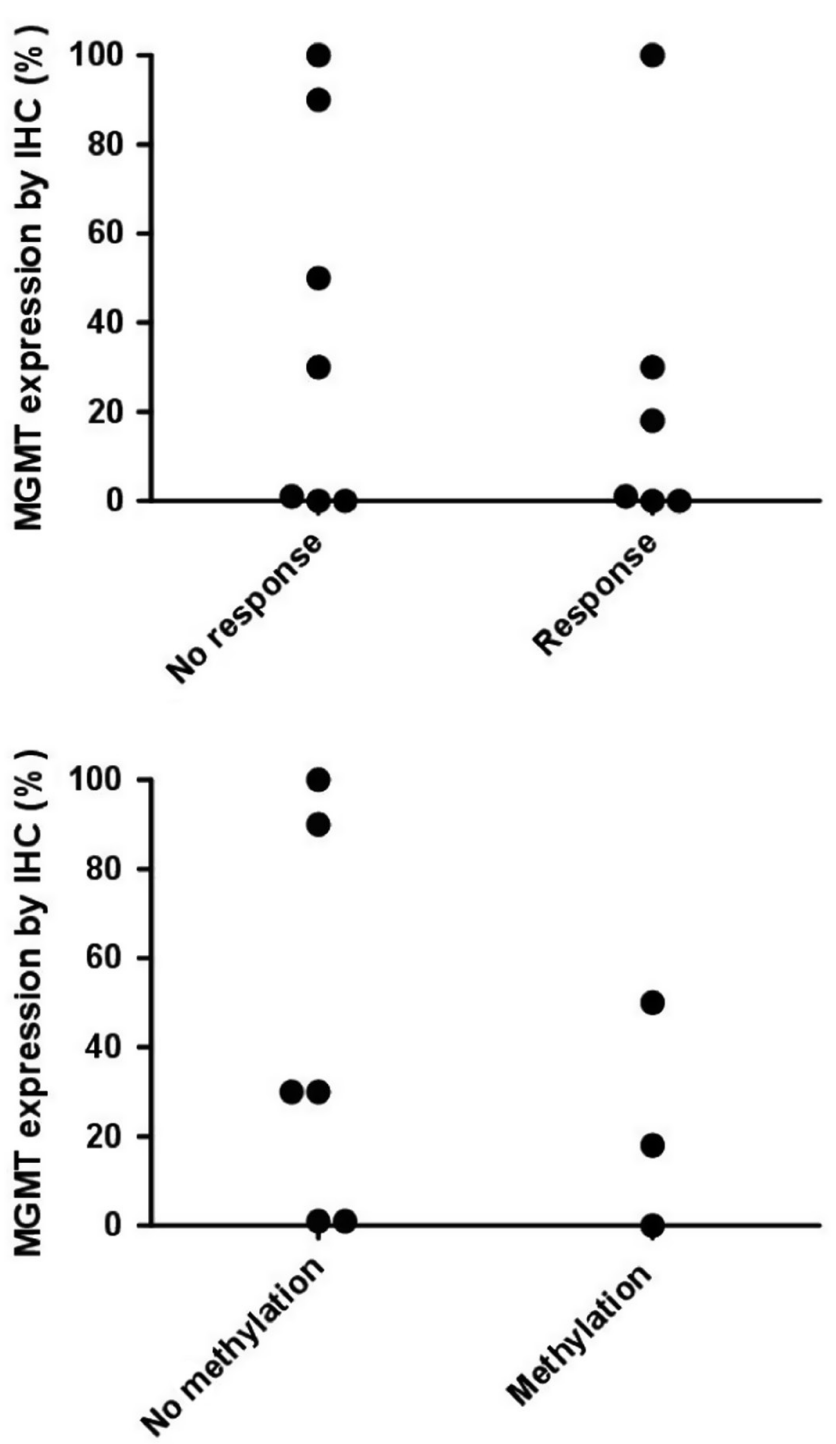

Figure 2

MGMT expression level according to TMZ hormonal and/or tumor response (13 patients) and MGMT expression according to promoter methylation (9 patients). 


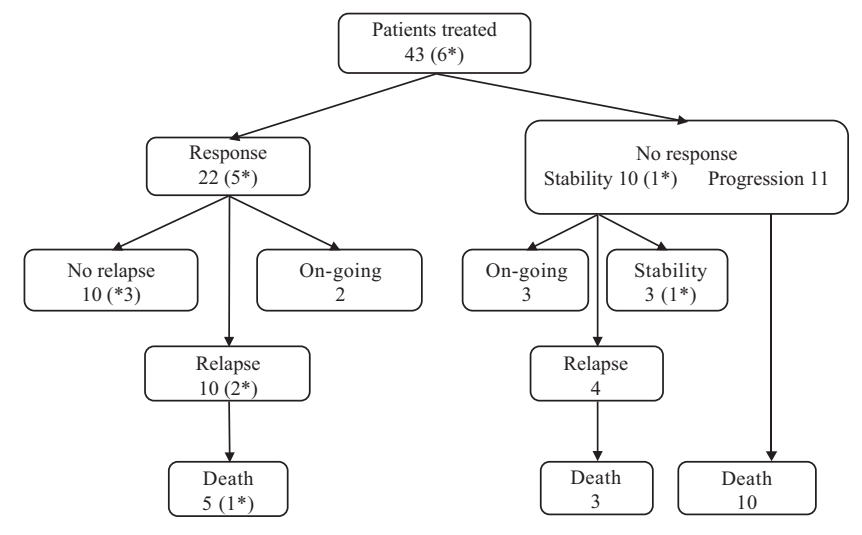

Figure 3

Flow-chart. *Whose $n$ received combined radio-chemotherapy protocol.

among non-responders ( $P$-value log-rank test=0.002) (Fig. 4B). This association was maintained in multivariate analysis $(\mathrm{HR}=0.08,95 \% \mathrm{CI}$ : $(0.02-0.38), P$-value Wald test $=0.002$ ). While increased age at TMZ initiation was associated with a lower survival, this effect was mild (HR for one year older $1.05, P$-value Wald test=0.01) and carcinomas compared to adenomas were at the limit of significance.

The median relapse-free survival post-TMZ treatment (in cases where it was deemed successful) was estimated at 30 months (18-51) (Fig. 4C). Among 19 patients with available follow-up, seven patients were treated with more than 12 cycles (median 18 cycles) and 12 with $\leq 12$ cycles. Patients with longer treatment periods may present a better relapse-free survival than patients with shorter treatment periods (medians of 57 and 18 months respectively, $P$-value log-rank $=0.07$ ). These 2 groups were comparable with regards to proportion of concomitant radiotherapy, Stupp protocol, tumor types and silent tumors. The proportion of carcinomas might be higher among patients treated $>12$ cycles compared to $\leq 12$ cycles (43\% vs $8 \%$ respectively), and patients with longer treatment periods also preferentially presented tumor response (100\% vs 75\% respectively) though this did not reach statistical significance. These factors were not associated with relapse-free survival ( $P$-value log-rank tests 0.85 and 0.78 respectively).

\section{Second line treatment}

After TMZ failure or tumor relapse (28 patients), another TMZ treatment was attempted for seven patients (including five initial responders and two
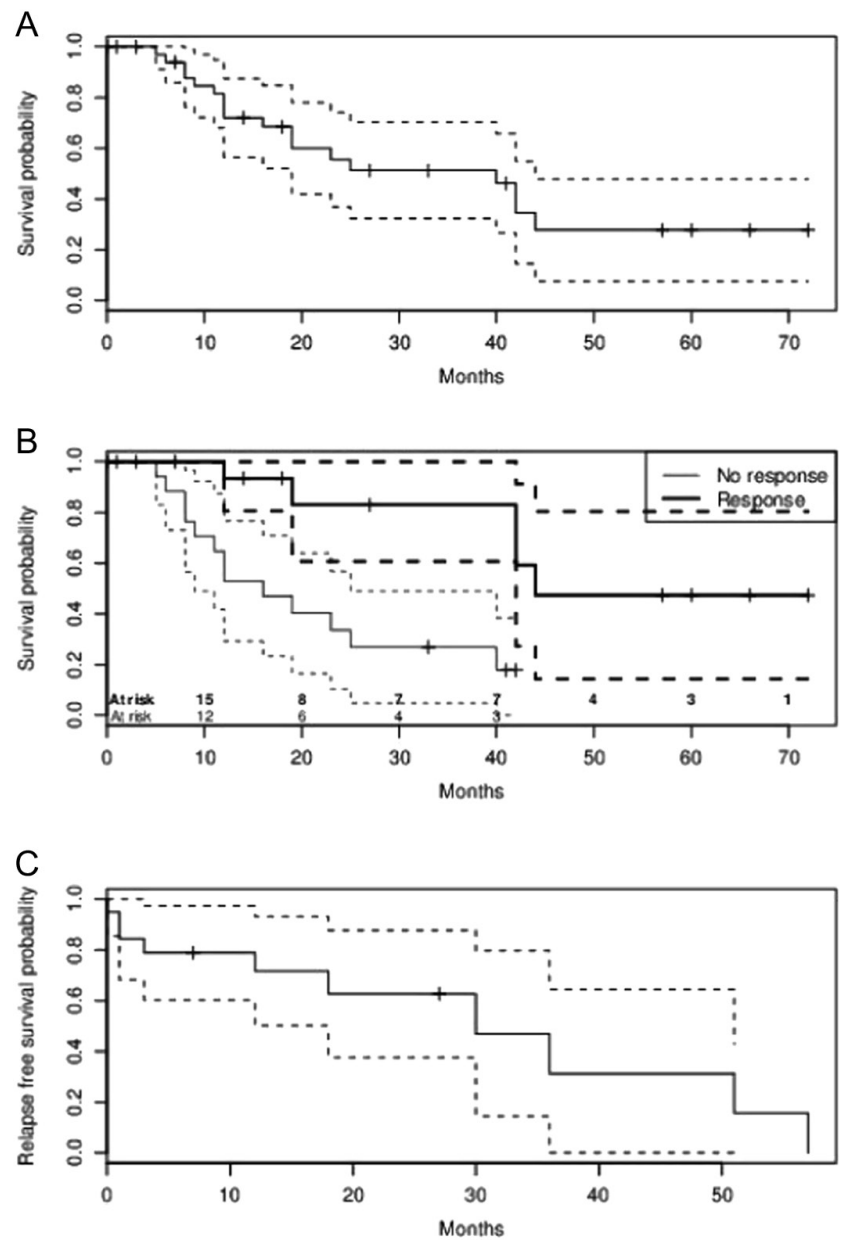

Figure 4

(A) Overall survival probability after TMZ end estimated with the Kaplan-Meier method (38 patients). (B) Overall survival probability after TMZ end according to response status, estimated with the Kaplan-Meier method in 20 responders and 18 non-responders. (C) Relapse-free survival probability after TMZ end among responders, estimated with the Kaplan-Meier method in 20 responders.

non-responders) though failed in all. This occurred two (0-7) years after the first TMZ attempt and involved three (1-5) cycles. Other treatments consisted of anew surgical excision $(n=10$, anew radiotherapy $(n=10)$, pasireotide $(n=4)$ and salvage therapies $(n=15)$. All salvage therapies (lomustine, carmustine, cyclophosphamide, etoposide $n=2$, carboplatin $n=2$, adriblastine, oxaliplatin-5FU, capecitabine, everolimus $n=3$, cisplatin $n=2$, lapatinib $n=2$, crizotinib, erlotinib, sunitinib) failed. Metabolic radiotherapy (DOTATOC) failed for one patient and is ongoing for another.

Among these 28 patients, only four (three showing stable responses to $\mathrm{TMZ}$ and one hormonal relapse) 
presented a stable disease at the last follow-up, all of whom received combined radiotherapy $(n=1)$ or adjuvant radiotherapy post-TMZ treatment $(n=3)$.

\section{Discussion}

This retrospective cohort of patients treated with TMZ for PT is to our knowledge the largest yet published work. It is highly representative of clinical practice and highlights the lack of a standardized protocol.

We have reported a prolonged follow-up after TMZ termination and observed that response to treatment strongly influenced the survival. Losa et al. observed a survival rate of $83.9 \%$ at two years and $59.6 \%$ at four years (14). Comparison between studies is difficult. In the Losa et al. study follow-up started after TMZ initiation. In our study in an attempt to limit bias in survival comparison produced by any relationship between treatment duration and response, we started the follow-up after treatment end. A 50.3\% survival rate could be estimated at 3 years after TMZ end, which was higher among responders than non-responders (median survival of 44 (42-infinity) and 16 (9-25) months respectively). However, half the patients (50\%) who responded well to treatment presented a relapse, which happened 15 (0-57) months after TMZ end. Those who did not relapse were followed up for only 9.5 (0-27) months; a longer follow-up might have revealed a higher rate of relapse.

The response rate (51.2\%) is in agreement with the recent cohort published by Bengtsson et al. with similar response criteria (15). Comparison to other published studies is difficult due to considerable differences in the response criteria across the studies $(14,19,20,21)$, and highlights the need for their standardization in order to better evaluate the treatment. Here, we decided to consider significant hormonal decrease as a response. If only tumor shrinkage were to be considered in our cohort, the response rate would have been $39.5 \%$. Some authors considered stability as a response (14, 20,21 ) but we only considered a significant reduction of the largest diameter to avoid over-estimating the response rate. The retrospective nature of the data evaluated in our multicenter study limited access to every pretreatment tumor growth curve. A stable course during the treatment could thus have been falsely interpreted as a response if the tumor was already stable before treatment. Moreover, pituitary tumors are often slow growing and 3-6 months may be too short to observe a significant growth of a potentially progressive tumor, especially of the largest diameter. However, tumor stabilization was observed in association with hormonal rate decrease for one patient although their tumor was rapidly progressive on monthly MRI before TMZ initiation.

The only baseline characteristic associated with response was initially silent tumors which appeared to be predictive of a poor response to TMZ. Among twelve tumors that were silent at diagnosis, seven (6 ACTH and 1 $\mathrm{GH})$ became secreting before TMZ treatment. Diagnosis of secretion was made based on clinical symptoms and was associated with tumor recurrence in all. Only two out of the 12 patients presented a response to TMZ treatment, which was hormonal, and seven out of the 12 died during follow-up. The median survival was 33 months among the patients with secondarily secreting tumors (5 deaths) and 12 months among those with tumors that remained silent ( 2 deaths); however, the difference did not reach statistical significance.

Neither MGMT expression nor its promoter methylation was predictive of response in our study. In contrast, in their centralized analysis of 17 tumors, Bengtsson et al. found significantly lower levels of MGMT staining in responders (median 9\% (5-20)) than in nonresponders (median 93\% (50-100)) (15). Although MGMT expression measurement was not centralized in our study, we did evaluate MGMT staining for 13 patients and found a median expression of 9.5\% (range (0-100)) among responders and 30\% (0-100) among non-responders with no significant difference between the two. Moreover, promoter methylation $(n=3 / 9)$ was not correlated with MGMT expression. While our study does report a noncentralized MGMT expression measurement which limits the conclusion on MGMT predictive value, it also represents real-life practice. MGMT analysis is not widely available and doubt remains over its prognostic value which may explain its absence in two thirds of our cohort and the physicians not considering it before initiating TMZ treatment. Finally, we assessed MGMT expression on the tumor fragment removed at the last surgery which in some cases was distant from TMZ treatment. In the 14 tumors with available MGMT analytical data (IHC and/or promoter methylation), the analysis had been performed on samples obtained 0-18 years before TMZ initiation. The analysis may not be representative of the tumor at the time of treatment.

The quite small number of patients and the retrospective nature of our study are the main limitations with regards evaluating predictive factors. In addition, an abundance of missing data disallowed a completely satisfactory analysis of histological features. 
In most cases reported in the literature, TMZ to treat PT was administered following the standard protocol used in gliomas i.e. $150-200 \mathrm{mg} / \mathrm{m}^{2} /$ day during 5 days every 28 days. There is a recent trend to associate the TMZ treatment with radiotherapy such as defined in the 'Stupp' protocol. While we are unable to provide evidence of its statistically significant benefit, the higher response rate $(83 \%)$ among the six patients who received this combined radio-chemotherapy should be underlined. A larger cohort is now needed to conclude on the potential benefit of this combined therapy; however, this initial result may encourage clinicians to consider this combined approach when TMZ treatment is indicated.

In accordance with previous studies, the early evaluation (after three cycles for most patients) was very predictive of the later response in our cohort $(15,18)$. The treatment duration was significantly shorter in nonresponders, showing a likely influence of early evaluation and clinician's choice in the decision to stop treatment, more than side effects (only one treatment cessation was due to side effects). As already suggested in the literature $(15,30)$, all anew treatment attempts with TMZ in cases of relapse failed, as did all salvage therapies. Therapeutic resources in such cases are therefore limited. The analysis of the sub-group of patients treated with more than 12 cycles suggests that extension of the treatment duration in responding patients could be an option in some instances. Conversely, a prolonged treatment in the event of no early response ( $n=2,18$ cycles) was never associated with a later response and exposes the patient to potential side effects.

In conclusion, we observed a successful treatment in about $50 \%$ of patients, associated with an important improvement in survival. Our study underlines the high risk of recurrence after cessation and more importantly the lack of tumor response in cases where TMZ was reintroduced. The benefits of concomitant radiotherapy and prolonged treatment have to be evaluated in larger studies. Finally, precise guidelines on TMZ indication and follow-up are needed to homogenize the practice among centers.

\section{Declaration of interest}

The authors declare that there is no conflict of interest that could be perceived as prejudicing the impartiality of this study.

\section{Funding}

This research did not receive any specific grant from any funding agency in the public, commercial or not-for-profit sector.

\section{Acknowledgements}

The authors thank Angloscribe for the scientific language editing and Olaf $M$ Dekkers for critically reviewing and commenting on the methodology.

\section{References}

1 Dolecek TA, Propp JM, Stroup NE \& Kruchko C. CBTRUS statistical report: primary brain and central nervous system tumors diagnosed in the United States in 2005-2009. Neuro-Oncology 201214 (Supplement 5) v1-v49. (doi:10.1093/neuonc/nos218)

2 Roelfsema F, Biermasz NR \& Pereira AM. Clinical factors involved in the recurrence of pituitary adenomas after surgical remission: a structured review and meta-analysis. Pituitary 201215 71-83. (doi:10.1007/s11102-011-0347-7)

3 Lloyd RV, Kovacs K, Young WF J, Farrell WE, Asa SL, Trouillas J, Kontogeorgos G, Sano T, Scheithauer BW \& Horvath E. Pituitary Tumours: Introduction. Lyon: IARC Press, 2004.

4 Tortosa F \& Webb SM. Atypical pituitary adenomas: 10 years of experience in a reference centre in Portugal. Neurología 201631 97-105. (doi:10.1016/j.nrleng.2015.06.003)

5 Trouillas J, Roy P, Sturm N, Dantony E, Cortet-Rudelli C, Viennet G, Bonneville JF, Assaker R, Auger C, Brue T et al. A new prognostic clinicopathological classification of pituitary adenomas: a multicentric case-control study of 410 patients with 8 years postoperative follow-up. Acta Neuropathologica 2013126 123-135. (doi:10.1007/s00401-013-1084-y)

6 Kaltsas GA, Nomikos P, Kontogeorgos G, Buchfelder M \& Grossman AB. Clinical review: diagnosis and management of pituitary carcinomas. Journal of Clinical Endocrinology and Metabolism 200590 3089-3099. (doi:10.1210/jc.2004-2231)

7 O'Reilly SM, Newlands ES, Glaser MG, Brampton M, Rice-Edwards JM, Illingworth RD, Richards PG, Kennard C, Colquhoun IR \& Lewis P. Temozolomide: a new oral cytotoxic chemotherapeutic agent with promising activity against primary brain tumours. European Journal of Cancer 1993 29A 940-942. (doi:10.1016/s0959-8049(05)80198-4)

8 Tatar Z, Thivat E, Planchat E, Gimbergues P, Gadea E, Abrial C \& Durando X. Temozolomide and unusual indications: review of literature. Cancer Treatment Reviews 201339 125-135. (doi:10.1016/j. ctrv.2012.06.002)

9 Stupp R, Mason WP, van den Bent MJ, Weller M, Fisher B, Taphoorn MJB, Belanger K, Brandes AA, Marosi C, Bogdahn U et al. Radiotherapy plus concomitant and adjuvant temozolomide for glioblastoma. New England Journal of Medicine 2005352 987-996. (doi:10.1056/NEJMoa043330)

10 Stupp R, Taillibert S, Kanner AA, Kesari S, Steinberg DM, Toms SA, Taylor LP, Lieberman F, Silvani A, Fink KL et al. Maintenance therapy with tumor-treating fields plus temozolomide vs temozolomide alone for glioblastoma: a randomized clinical trial. JAMA $2015 \mathbf{3 1 4}$ 2535-2543. (doi:10.1001/jama.2015.16669)

11 Lim S, Shahinian H, Maya MM, Yong W \& Heaney AP. Temozolomide: a novel treatment for pituitary carcinoma. Lancet Oncology 20067 518-520. (doi:10.1016/S1470-2045(06)70728-8)

12 Fadul CE, Kominsky AL, Meyer LP, Kingman LS, Kinlaw WB, Rhodes CH, Eskey CJ \& Simmons NE. Long-term response of pituitary carcinoma to temozolomide. Report of two cases. Journal of Neurosurgery 2006105 621-626. (doi:10.3171/jns.2006.105.4.621)

13 Syro LV, Uribe H, Penagos LC, Ortiz LD, Fadul CE, Horvath E \& Kovacs K. Antitumour effects of temozolomide in a man with a large, invasive prolactin-producing pituitary neoplasm. Clinical Endocrinology 200665 552-553. (doi:10.1111/j.13652265.2006.02653.x)

14 Losa M, Bogazzi F, Cannavo S, Ceccato F, Curtò L, De Marinis L, Iacovazzo D, Lombardi G, Mantovani G, Mazza E et al. Temozolomide therapy in patients with aggressive pituitary adenomas or carcinomas. 
Journal of Neuro-Oncology 2016126 519-525. (doi:10.1007/s11060015-1991-y)

15 Bengtsson D, Schrøder HD, Andersen M, Maiter D, Berinder K, Feldt Rasmussen U, Rasmussen ÅK, Johannsson G, Hoybye C, van der Lely AJ et al. Long-term outcome and MGMT as a predictive marker in 24 patients with atypical pituitary adenomas and pituitary carcinomas given treatment with temozolomide. Journal of Clinical Endocrinology and Metabolism 2015100 1689-1698. (doi:10.1210/ jc.2014-4350)

16 Bruno OD, Juárez-Allen L, Christiansen SB, Manavela M, Danilowicz K, Vigovich C \& Gómez RM. Temozolomide therapy for aggressive pituitary tumors: results in a small series of patients from Argentina. International Journal of Endocrinology 20152015587893.

17 Losa M, Mazza E, Terreni MR, McCormack A, Gill AJ, Motta M, Cangi MG, Talarico A, Mortini P \& Reni M. Salvage therapy with temozolomide in patients with aggressive or metastatic pituitary adenomas: experience in six cases. European Journal of Endocrinology 2010163 843-851. (doi:10.1530/EJE-10-0629)

18 Raverot G, Sturm N, de Fraipont F, Muller M, Salenave S, Caron P, Chabre O, Chanson P, Cortet-Rudelli C, Assaker R et al. Temozolomide treatment in aggressive pituitary tumors and pituitary carcinomas: a French multicenter experience. Journal of Clinical Endocrinology and Metabolism 201095 4592-4599. (doi:10.1210/jc.2010-0644)

19 Bush ZM, Longtine JA, Cunningham T, Schiff D, Jane JA, Vance ML, Thorner MO, Laws ER \& Lopes MBS. Temozolomide treatment for aggressive pituitary tumors: correlation of clinical outcome with $\mathrm{O}^{6}$-methylguanine methyltransferase (MGMT) promoter methylation and expression. Journal of Clinical Endocrinology and Metabolism 2010 95 E280-E290. (doi:10.1210/jc.2010-0441)

20 Hirohata T, Asano K, Ogawa Y, Takano S, Amano K, Isozaki O, Iwai Y, Sakata K, Fukuhara N, Nishioka H et al. DNA mismatch repair protein (MSH6) correlated with the responses of atypical pituitary adenomas and pituitary carcinomas to temozolomide: the national cooperative study by the Japan Society for Hypothalamic and Pituitary Tumors. Journal of Clinical Endocrinology and Metabolism 201398 1130-1136. (doi:10.1210/jc.2012-2924)

21 Ceccato F, Lombardi G, Manara R, Emanuelli E, Denaro L, Milanese L, Gardiman MP, Bertorelle R, Scanarini M, D'Avella D et al. Temozolomide and pasireotide treatment for aggressive pituitary adenoma: expertise at a tertiary care center. Journal of Neuro-Oncology 2015122 189-196. (doi:10.1007/s11060-014-1702-0)
22 Lasolle H \& Raverot G. Therapeutic innovations in endocrine diseases - part 3: temozolomide and future therapeutics for aggressive pituitary tumors and carcinomas. Presse Médicale 201645 e211-e216. (doi:10.1016/j.lpm.2016.05.008)

23 Cao VT, Jung T-Y, Jung S, Jin S-G, Moon K-S, Kim I-Y, Kang S-S, Park C-S, Lee K-H \& Chae H-J. The correlation and prognostic significance of MGMT promoter methylation and MGMT protein in glioblastomas. Neurosurgery 200965 866-875; discussion 875. (doi:10.1227/01.NEU.0000357325.90347.A1)

24 Raverot G, Castinetti F, Jouanneau E, Morange I, Figarella-Branger D, Dufour H, Trouillas J \& Brue T. Pituitary carcinomas and aggressive pituitary tumours: merits and pitfalls of temozolomide treatment. Clinical Endocrinology 201276 769-775. (doi:10.1111/j.13652265.2012.04381.x)

25 Whitelaw BC, Dworakowska D, Thomas NW, Barazi S, Riordan-Eva P, King AP, Hampton T, Landau DB, Lipscomb D, Buchanan CR et al. Temozolomide in the management of dopamine agonist-resistant prolactinomas. Clinical Endocrinology 201276 877-886. (doi:10.1111/ j.1365-2265.2012.04373.x)

26 Jouanneau E, Wierinckx A, Ducray F, Favrel V, Borson-Chazot F, Honnorat J, Trouillas J \& Raverot G. New targeted therapies in pituitary carcinoma resistant to temozolomide. Pituitary 201215 37-43. (doi:10.1007/s11102-011-0341-0)

27 Batisse M, Raverot G, Maqdasy S, Durando X, Sturm N, Montoriol PF, Kemeny JL, Chazal J, Trouillas J \& Tauveron I. Aggressive silent GH pituitary tumor resistant to multiple treatments, including temozolomide. Cancer Investigation 201331 190-196. (doi:10.3109/07 357907.2013.775293)

28 Zemmoura I, Wierinckx A, Vasiljevic A, Jan M, Trouillas J \& Francois P. Aggressive and malignant prolactin pituitary tumors: pathological diagnosis and patient management. Pituitary 201316 515-522. (doi:10.1007/s11102-012-0448-y)

29 Philippon M, Morange I, Barrie M, Barlier A, Taieb D, Dufour H, Conte-Devolx B, Brue T \& Castinetti F. Long-term control of a MEN1 prolactin secreting pituitary carcinoma after temozolomide treatment. Annales d'Endocrinologie 201273 225-229. (doi:10.1016/j. ando.2012.03.001)

30 Campderá M, Palacios N, Aller J, Magallón R, Martín P, Saucedo G, Lilienfeld H \& Estrada J. Temozolomide for aggressive ACTH pituitary tumors: failure of a second course of treatment. Pituitary 201619 158-166. (doi:10.1007/s11102-015-0694-x)

Received 28 November 2016

Revised version received 20 March 2017

Accepted 27 March 2017 